\title{
DOCTRINA
}

\section{Deporte, prueba y decisión arbitral: Sobre la asistencia en video}

\author{
Sport, and referee decision: About video assistance
}

\section{Jesús Ezurmendia Álvarez (D) y Jonatan Valenzuela Saldías \\ Universidad de Chile}

\begin{abstract}
RESUMEN En el presente artículo se pretende utilizar el fútbol, y en especial la incorporación de asistencia de video en la toma de decisiones en juego, como herramienta para explicar las ideas básicas de la teoría de la prueba en el derecho. Dentro de lo que se trata, se incluyen los momentos de la actividad probatoria, el objeto de prueba y los estándares y cargas probatorias, así como la legitimación y la iniciativa para la verificación de supuesto fáctico. Se presenta como una forma de relacionar contenidos teóricos del derecho probatorio y la epistemología jurídica en el contexto de deportes masivos, en que su utilización parece más cercana a quienes inician su estudio de la evidencia desde una perspectiva teórica.
\end{abstract}

PALABRAS CLAVE Derecho procesal, derecho probatorio, prueba y deporte, tecnología.

ABSTRACT This article aims to use soccer, and especially the incorporation of video assistance in decision-making at play, as a tool to explain certain elements and institutions of the basic ideas of the theory of evidence in law. What is covered are the moments of the evidentiary activity, the proof's object, the standards and evidentiary burdens as well as the initiative to request the verification of a factual situation. It aims to enhance the link between abstract contents of evidence legal theory and legal epistemology in the context of mass sports, in which their utilization seems to be relatable for those who initiate their studies of evidence from a theoretical perspective.

KEYWORDS Procedural law, evidence law, sports and evidence, technology. 


\section{Introducción: El fútbol como herramienta metodológica}

«El fútbol es el deporte más hermoso del mundo», expresó Eduardo Galeano en Fútbol a sol y a sombra. Cada partido genera una especie de encanto ineludible y parecen ser muchos los que dedicamos considerable tiempo de nuestras vidas a jugar al fútbol y mirarlo. ${ }^{1}$ En términos coloquiales, el equipo de fútbol de un sujeto es una muestra de su comprensión del mundo y la de quienes le rodean. Cambiar de equipo de fútbol es, probablemente, uno de los actos de alta traición más importantes, por lo que simboliza. Es probable que las decisiones adoptadas en ese marco son tan importantes que pueden ser fácilmente recordadas por la enorme mayoría de personas en el mundo.

El fútbol, como el proceso, es un escenario de producción de decisiones. Cada lunes en las oficinas, los colegios, las universidades y la calle se comentan en detalle cada decisión arbitral en torno al juego. La regla del offside o posición adelantada, la valoración de ciertos movimientos, la intencionalidad al tocar el balón con la mano, entre muchos otros tópicos, son objeto de largas conversaciones. Así visto, consideramos que gran parte de las discusiones que se producen en el ámbito futbolístico valen como una clase de contexto en que el razonamiento probatorio puede ser examinado.

Ese examen puede producirse, al menos, desde dos perspectivas. Podríamos pensar en las decisiones empíricamente consideradas y evaluar el razonamiento que tiene un árbitro o los árbitros de una determinada comunidad respecto de ciertos puntos propios del razonamiento probatorio. Debe considerarse que estas decisiones son marcadamente inmediatas y orales. Es decir, son decisiones que se producen ante los hechos que deben juzgarse, de manera oral y con pretensiones de evitar por completo la mediación de las partes. Por otro lado, podríamos evaluar alguna institución o las reglas sobre la prueba en un determinado contexto que justifica decisiones desde el punto de vista probatorio. En esta perspectiva, la manera en que las decisiones son adoptadas cobra relevancia, frente a reglas que definen el modo en que los árbitros del juego pueden justificar la concurrencia de la premisa fáctica de sus decisiones.

En este trabajo, quisiéramos centrar nuestra atención en el video assistant referee (VAR), el asistente en video de los árbitros del fútbol, sistema que ha generado una gran atención como mecanismo reductor de errores al permitir, en teoría, evitar cobros errados. Pretendemos utilizar el fútbol, y en especial la incorporación de asistencia de video en la toma de decisiones en juego, como herramienta para explicar ciertos elementos e instituciones de la teoría de la prueba. Dentro de lo que se trata

1. La última final del Campeonato Mundial de Fútbol fue seguida por televisión por 3.572 millones de personas, la mitad de la población mundial, según datos publicados por la Federación Internacional de Fútbol Asociado (FIFA). «Más de la mitad del planeta disfrutó de un Mundial incomparable en 2018», FIFA.com, 21 de diciembre de 2018, disponible en https://fifa.fans/3ncmvAY. 
se incluyen los momentos de la actividad probatoria, el objeto de prueba y los estándares y cargas probatorias.

Creemos que, en ese escenario, la discusión sobre naturaleza del VAR desde el punto de vista probatorio para revisar algunos aspectos importantes de las decisiones de árbitros de fútbol resulta llamativa.

Además, el uso de un escenario de decisiones como el fútbol permite introducir a los lectores en el razonamiento probatorio. La aplicación de contextos más cercanos y comunes para quien se inicia en el estudio de una determinada disciplina suele ser uno de los recursos metodológicos más comunes en el campo de las ciencias sociales. Así, el derecho recurre a estas herramientas para explicar algún fenómeno de estudio que puede presentarse lejano al estudiante, de forma de aproximarse de una forma más amigable al tópico.

En la literatura de la teoría del derecho existen ejemplos célebres, como el de Lon Fuller en su conocido caso de los exploradores (Fuller, 2002), que ha sido utilizado para explicar el derecho natural, la ética kantiana e incluso los derechos humanos (Schauer, 2016: 11). A su vez, dentro de los contextos de mejor asimilación por el lector general, las comparaciones y analogías deportivas, como la que presentamos en este ensayo, han resultado en particular útiles y las de mejor aceptación por parte del destinatario de la explicación, quien ve desde un contexto conocido, familiar e incluso lúdico un acercamiento a la disciplina nueva. En este sentido, resulta excepcional la explicación de la regla de reconocimiento que H. L. A. Hart ensaya al comparar las normas con las reglas de anotación de goles o tantos de un juego o deporte (Hart, 1994: 102). ${ }^{2}$

\section{Deporte y teoría de la prueba}

En este trabajo, quisiéramos introducir ciertos problemas de la teoría de la prueba y el derecho probatorio, utilizando la relevancia cultural del fútbol y otros deportes profesionales como catalizadores. Lo antedicho tendrá como telón de fondo uno de los postulados más significativos desde el cognoscitivismo crítico, y es que la actividad probatoria que se desenvuelve en el contexto de un proceso judicial no es ajena ni radicalmente distinta a cualquier otro contexto probatorio (Ferrer, 2005: 69-78).

El fútbol tampoco es ajeno a estas reglas comunes de racionalidad epistémica de reconstrucción de hechos o de generación de conocimiento. Así, en este trabajo intentaremos formular una descripción de ciertas cuestiones relativas a la teoría de la prueba y al derecho procesal en general, como las reglas de exclusión, la aplicación de estándares de prueba e incluso la cosa juzgada, explicadas a través de decisiones to-

2. Este pasaje incluso ha sido tildado como el más célebre de su obra The concept of law (Russel, 2011: 254). 
madas por agentes reglamentarios - jueces, árbitros, umpires - durante encuentros deportivos en los que existe revisión de jugadas específicas a través de asistente de video, cuya aplicación ha aumentado en los últimos años. Esta posibilidad de revisión de decisiones tomadas dentro de la cancha en el desarrollo del juego permite, asimismo, situarse en el contexto análogo de la impugnación de las decisiones judiciales, o incluso la revisión inquisitiva de éstas en el contexto del proceso judicial, en especial en lo referido a la aplicación de una regla general a un supuesto de hecho específico que es posible revisar por completo.

La revisión por medio de herramientas tecnológicas de decisiones tomadas por jueces deportivos se construye en un determinado marco institucional (Bayon, 2010, 7), en el que se regulan una serie de aspectos acerca de la corroboración de los hechos, lo que evita que la actividad se utilice en forma arbitraria y abusiva, lo cual atentaría contra los propios objetivos de su instauración.

El árbitro asistente de video ( $\mathrm{AAV}$, más conocido como VAR, por sus siglas en inglés) comenzó a utilizarse durante 2017 en competiciones de la Federación Internacional de Futbol Asociación (FIFA). Desde sus orígenes se ha discutido la aplicabilidad de la tecnología como garantía para obtener una mejor decisión por parte de los árbitros sobre jugadas determinantes. ${ }^{3}$

Este mecanismo, incluso, fue incorporado en el Mundial de Rusia $2018^{4} \mathrm{y}$, por medio de una práctica bastante discutible, en la última Copa América de Brasil 2019. ${ }^{5}$

Sin perjuicio de la repercusión mediática que procede de la caja de resonancia del fútbol, ${ }^{6}$ el VAR no representa el primer intento de utilización de evidencia videograbada en tiempo real para asistencia en decisiones dentro del juego. En deportes menos populares a nivel mundial, en particular algunos de gran difusión en países angloparlantes, la revisión de video para corregir una decisión ha sido aplicada durante años. ${ }^{7}$

En aquellos deportes en que es aplicable la asistencia de video para mejorar la toma de decisión, existe una reglamentación más o menos acuciosa de determinadas cuestiones que suponen normar la compatibilidad del cobro correcto o acertado con

3. «Reglas del juego 2017/2018», International Football Association Board (IFAB), p. 12, disponible en https://bit.ly/2Imov7B.

4. Richard Conway, «VAR: Video assistant referees set to be used at 2018 World Cup in Russia», BBC. com, Sports, 3 de marzo de 2018, disponible en https://bbc.in $/ 3 \mathrm{kglpSz}$.

5. Véase sobre su uso «Las grandes polémicas del VAR en la Copa América de Brasil 2019», Cooperativa, Al Aire Libre, 8 de julio de 2019, disponible en https://bit.ly/3eKo56Q.

6. El futbol se erige como la disciplina deportiva más famosa a nivel mundial, la que mayores ingresos genera a nivel de derechos de imagen y el contenido más vistos en todo tipo de plataformas (Nauright y Zipp, 2018: 1.115-1.116).

7. Es posible encontrar reglas sobre el tema en la National Football League (NFL), la National Basketball Association (NBA) y el International Rugby Board (IRB), entre otros. 
la continuidad propia del juego (Calandrillo y Davison, 2017: 14), la cual es entendida por todas las asociaciones deportivas como un ámbito central del desarrollo de la actividad, que debe ser respetado e incluso preferido ante la solución "correcta» desde el punto de vista probatorio (Calandrillo y Davison, 2017: 1).

Así, en asociaciones como la National Football League (NFL) y la National Basketball Asociation (NBA) en Estados Unidos, la Federación Internacional de Básquetbol Asociación (FIBA) y la International Rugby Board (IRB) a nivel mundial, es posible encontrar que la revisión de video está normada en atención a los siguientes criterios:

- Se establece expresamente qué jugadas o cobros arbitrales pueden ser objeto de revisión, es decir, se delimita un ámbito de aplicación de la revisión. ${ }^{8}$

- Se establece una oportunidad para la revisión que, por lo general, suele estar limitada para impedir la excesiva interrupción del juego, y respecto de la que es posible encontrar mayor aplicabilidad en momentos decisivos o finales del encuentro. ${ }^{9}$

- Se establece la iniciativa para solicitar la revisión, señalándose si corresponderá al árbitro o árbitros de oficio, si corresponde solicitarla al equipo interesado, o si, por el contrario, es una regla de aplicación automática para toda jugada o cobro que se cumpla con determinadas características descritas por la norma. ${ }^{10}$

- Se ha establecido un estándar de prueba que debe ser superado por parte de la evidencia en video para determinar la decisión final. ${ }^{11}$

De esta manera, se trata de un sistema que comparte muchos criterios asociados prima facie al contexto judicial, toda vez que existe: i) una regla de aplicabilidad; ii) una regla de objeto; iii) una regla de oportunidad; iv) una regla de legitimación; y v) una regla de estándar (Calandrillo y Davison, 2017: 1), la que además va asociada a una regla de carga o presunción, como se expresa a continuación.

\section{Objeto de prueba y contenido de la revisión: Lo «revisable»}

Dentro del contexto judicial de la prueba, una de las cuestiones de mayor relevancia es establecer el objeto de prueba. Por objeto de prueba tradicionalmente se ha enten-

8. «2017 NFL Rulebook», regla 15, sección 2, artículo 1, disponible en https://bit.ly/308sUh5; 2013-2014 NBA Rulebook, regla 13, disponible en https://on.nba.com/33j6a5N; «2016 FIBA Procedure for the application of the Instant Replay System (IRS)», p. 5, disponible en https://bit.ly/3nVKUuW; «Leyes del juego de rugby, World Rugby, disponible en https://bit.ly/35eNCFg.

9. 2017 NFL Rulebook, regla 15, sección 2, artículo 1.

10. 2017 NFL Rulebook, regla 15, sección 2, artículo 1.

11. 2017 NFL Rulebook, regla 15, sección 2, artículo 1. 
dido la materia o temática sobre la que recae la actividad probatoria (Ortells, 2010: 353), es decir, aquello que debe probarse (Couture, 1981: 219) o, como señalan Maturana y Montero (2012: 837), «aquello sobre lo cual puede verificarse demostración en el proceso».

No existe duda de que debe tratarse de cuestiones fácticas, por oposición a aquellas cuestiones de derecho sobre las que, en virtud de la ficción de conocimiento de la ley y el conocimiento que de la misma debe tener el juez, no deberá recaer probanza alguna. Hay dudas, en cambio, en qué es lo que queremos señalar cuando decimos «cuestiones fácticas».

Que la prueba deba recaer sobre cuestiones de hecho no significa que toda cuestión fáctica vaya a ser sometida a verificación en el proceso. Así, habrá enunciados sobre los hechos que no serán controvertidos, o que recaerán sobre hechos evidentes o notorios (Maturana y Montero, 2012: 851-855), o que por ser parte del acervo cultural de los intervinientes o responder a conocimientos científicos conocidos por el juez, no deberán ser probados. ${ }^{12}$ Se trata de cuestiones de contenido fáctico, pero respecto de las cuales no será necesario iniciar proceso alguno de verificación. Las reglas de revisión de decisiones deportivas establecen ciertas limitaciones o restricciones respecto de cuestiones fácticas que no podrán ser objeto de prueba. Se trata de enunciados que, pudiendo ser disputados y relevantes para el resultado de la contienda, han sido sustraídos de la posibilidad de ser revisados. En este caso, la imposibilidad de revisión no es consecuencia del conocimiento general o inferencial que pueda existir sobre los hechos, sino que existe una barrera normativa que ha restringido la posibilidad de revisitar la decisión, la que se materializa — por regla general- a través del establecimiento de listas numerus clausus de decisiones revisables, una suerte de catálogo de hechos o supuestos a probar factum probandum. De esta manera, por ejemplo, el VAR es aplicable, de acuerdo con los principios señalados en el protocolo de prueba de la IFAB, sólo a cuatro posibles decisiones arbitrales: i) goles (incluida la posición adelantada que le sirve de antecedente); ii) decisiones de tiro penal; iii) tarjetas rojas directas; y iv) errores de identificación. ${ }^{13}$ Es decir, puede someterse al VAR una duda sobre si una jugada es o no es gol, a si corresponde una tarjeta roja directa, a si una jugada es o no penal y a la identificación de un determinado jugador.

Con esta nómina de decisiones revisables, se establece que será objeto de prueba videoasistida sólo aquella jugada que pueda enmarcarse dentro de las cuatro hipótesis de la norma. De forma similar ha sido limitada la revisión en la regla 13 del libro de reglas de la NBA en las secciones 1 y 2, y en la NFL en la regla 15, sección 2, artículo 5.

12. En el derecho del Common Law este tipo de hechos que no son objeto de pruebas se agrupan bajo la doctrina del judicial notice (Zuckerman, 2013: 1.036; Keane y Mckeown, 2014: 690-691).

13. «Protocolo sobre experimento con árbitros asistentes de vídeo (AAV)», International Football Association Board, disponible en https://bit.ly/36uooDU. 
Cabe hacer presente que en los procesos judiciales la determinación del objeto de prueba en concreto es resorte del juez, por lo que es éste - por lo general a través de una resolución-quien fija los hechos sobre los cuales deberá recaer la actividad probatoria. En las decisiones deportivas la regla general es la misma, aunque la fijación de lo que será revisable en el caso particular se hace siempre en el marco de lo que la norma de aplicación de la revisión por video permite revisar, es decir, dentro de los supuestos de revisión previstos por la regla, el juez puede determinar que una jugada específica se revise. La excepción a esta regla está dada por algunas organizaciones, como la NFL, en las que ciertas y determinadas jugadas, como las anotaciones, deben necesariamente ser revisadas por mandato de las propias reglas, sin que haya una fijación discrecional del cuerpo arbitral (regla 15, sección 2, artículo 5).

Esta limitación supone una decisión normativa del cuerpo, institución o ente con facultades regulatorias generales, en atención a qué cuestiones de hecho serán o no objeto de revisión, lo que constituye en muchos casos verdaderos límites de acceso al conocimiento efectivo sobre lo ocurrido.

Tomemos como ejemplo lo dispuesto en el protocolo de la IFAB respecto de la restricción a la revisión de infracciones que originen una segunda tarjeta amarilla. ${ }^{14} \mathrm{Se}$ trata de una jugada que - en consecuencia - terminará con la expulsión del infractor, y sobre la que podría existir una duda importante. Sin embargo, no será revisable, mientras que si fuere una tarjeta roja directa sí lo sería, incluso si la infracción es de una gravedad tal o se produce en circunstancias que - en el evento de ser efectivapodría originar una tarjeta roja o amarilla. En tal caso, el árbitro, en el supuesto de conceder la infracción y aplicar la sanción de la tarjeta amarilla, no quedaría dentro de los supuestos de aplicación del VAR, lo que le impediría revisar su decisión, aun si el propio juez tiene duda.

\section{Iniciativa y legitimación para solicitar la revisión}

Uno de los aspectos más relevantes de la prueba en el contexto judicial —también llamado «rasgos procesales de la asunción de la prueba» (Taruffo, 2008: 109) — está dado por la iniciativa para su producción y presentación, es decir, para definir qué sujetos de aquéllos que intervienen en el proceso están facultados para incorporar evidencia que sirva para corroborar una determinada hipótesis sobre los hechos.

Suele señalarse que existen dos grandes sistemas que orbitan el rol que le cabe al juez en la producción de prueba (Taruffo, 2008: 109). Por un lado, existen sistemas adversariales - centrados en las partes-, en los cuales el rol del sentenciador es pasivo: no está permitido el impulso inquisitivo u oficial en lo referido a materias de

\footnotetext{
14. IFAB. «Protocolo...», 5.
} 
prueba, e incluso de manera más general, el juez está inhibido de hacer cualquier actuación que no haya sido provocada o solicitada por las partes (Taruffo, 2008: 109).

Existen también sistemas, tradicionalmente asociados al sistema civil o continental, en que el rol del juez es indispensable en la averiguación de los hechos, por lo que no sólo autorizado, sino que muchas veces obligado a proponer y producir evidencia, independiente de las solicitudes que hagan las partes interesadas. Según Taruffo, estos sistemas se denominarían «centrados en el tribunal» (Taruffo, 2008: 109).

Cabe hacer presente que ninguno de estos sistemas se presenta hoy en día, al menos en materia de proceso civil, en forma completamente pura, toda vez que los sistemas de justicia han encontrado formas intermedias o eclécticas, basados en la preponderancia de ciertos principios o reglas por sobre otras (Taruffo, 2008: 109), que permiten describir un sistema como más cercano a un extremo que al otro del péndulo, pero en ningún caso como una aceptación total de uno u otro modelo.

En ese contexto, resulta explicativo señalar que en materia de revisión videoasistida de decisiones deportivas durante el juego, existen sistemas que permiten la revisión del fallo a exclusiva iniciativa de los interesados, otros sistemas en los que es exclusiva prerrogativa del juez, y otros en los que algunos cobros deben - cumplidos ciertos requisitos- ser revisados por mandato expreso de la norma, sin que haya una decisión de ninguno de los involucrados a tal respecto.

En ninguna de las organizaciones de deportes colectivos de mayor relevancia existe un sistema en que la revisión esté por completo entregada a los equipos (partes), sino que existen sistemas exclusivamente oficiales o mixtos. Dentro de los deportes masivos de mayor popularidad en el mundo, la NFL cuenta con el sistema intermedio, en el que la posibilidad de revisión de fallos arbitrales está entregada a los equipos y a los propios jueces. Así, en un juego se le entrega a cada equipo, representado por el entrenador jefe, la posibilidad de desafiar dos cobros que - revisables según materia - a su juicio deben ser objeto de revisión. Además, si ambas revisiones resul$\tan$ exitosas, se le concede una tercera oportunidad. ${ }^{15}$

Por su parte, los árbitros tienen una iniciativa más amplia, pues existe la posibilidad de revisión oficial, por parte del cuerpo arbitral, de cualquier jugada dentro de los últimos dos minutos de cada mitad del juego, así como durante el tiempo suplementario. Para ello, no existe un número determinado de revisiones, que pueden ser tantas como se consideren necesarias. A su vez, se establece una revisión automática, distinta a aquella iniciada de oficio por los árbitros, para las jugadas más relevantes del juego, como las anotaciones e intercepciones.16 Debe distinguirse, en consecuencia, esta última de la iniciativa de oficio por los árbitros, toda vez que no existe un ejercicio discrecional de los jueces, o la existencia de una duda que les haga solicitar

15. 2016 NFL Rulebook, regla 15, sección 2, artículo 1.

16. 2016 NFL Rulebook, regla 15, sección 2, artículo 1. 
la revisión, sino una regla que obliga a la revisión por parte de los árbitros, independiente de su voluntad al respecto.

Por su parte, la NBA ha optado por un sistema mixto de iniciativa automática y oficial. En el baloncesto profesional de Estados Unidos la revisión de las jugadas según la materia hay quince posibles objetos de revisión- puede, en primer término, ser instantánea y obligatoria para el caso de ciertas circunstancias dentro del juego que revisten una especial dificultad en su sanción, como una anotación ocurrida cuando no quedaba tiempo en el reloj del respectivo periodo o el reloj de posesión. ${ }^{17}$ En tales casos, la revisión es obligatoria para los jueces. Las demás sanciones, cobros y jugadas que no estén incluidas en el catálogo de revisión automática sólo podrán ser revisados a iniciativa de los árbitros siempre que cualquiera de ellos no esté «razonablemente seguro» (not reasonable certain) sobre lo que en efecto ha ocurrido. ${ }^{18}$

Éste es un caso de sistema de iniciativa inquisitiva, en el que lo que hace posible la decisión es la existencia de incertidumbre entre los jueces, la que ha de ser, además, razonable. En este sistema les está privado a los interesados (los equipos) solicitar la revisión de cualquier cobro, sin importar lo inexacto que les haya parecido.

En el caso del fútbol, el protocolo IFAB para aplicación del VAR regula la iniciativa de la asistencia en video a los réferis. Al respecto, en el numeral sexto del acápite «Principios» se señala que: «Solamente el árbitro puede iniciar una revisión; el AAV (y los otros miembros del equipo arbitral) solamente pueden recomendar una revisión al árbitro» ${ }^{19}$ con lo que establece de forma clara un sistema de revisión exclusivamente inquisitivo, pero además discrecional, en el que la revisión de una jugada —de aquéllas que puede revisarse según el mismo protocolo- es una facultad entregada en exclusiva al árbitro central del partido.

Desde el punto de vista de la iniciativa, entonces, es posible señalar que en todos los sistemas que han regulado la revisión de fallos dentro de partidos, los jueces o árbitros tienen poderes inquisitivos para utilizar la herramienta. Lo que ha tenido dispar recepción ha sido la decisión de entregar iniciativa a las partes para dicha asistencia audiovisual; sin embargo, aun en los casos en que se permite, la revisión oficial suele ser más amplia y con mayores facultades que aquélla entregada a los equipos participantes.

Lo anterior permite señalar que se regulan a través de una norma inversa a la que suele ser utilizada en contextos procesales, pues la regla general - como se ha establecido- es que la iniciativa siempre la tengan las partes que pretenden demostrar la veracidad de sus enunciados, mientras que las facultades probatorias inquisitivas de los jueces son excepcionales o, al menos, sustancialmente menos comunes y más restringidas que las de los demás intervinientes (Taruffo, 2008: 164-171).

17. 2013-2014 NBA Rulebook, regla 13, sección 1.

18. 2013-2014 NBA Rulebook, regla 13, sección 1, números 6, 7, 8, 10, 11 y 12.

19. IFAB, «Protocolo...», 6. 


\section{Reglas contraepistémicas, en especial reglas de exclusión de evidencia}

La prueba jurídica no es, en realidad, distinta de la prueba como actividad general del razonamiento. Sin embargo, la existencia de reglas que limitan el conocimiento en atención a la protección de valores puede encontrarse con particulares características en el ámbito jurídico.

Se trata de limitaciones a dicha actividad que impiden un despliegue completo y absoluto de actividad de corroboración de los enunciados fácticos, fundada en la existencia de valores diversos a la averiguación de la verdad que el ordenamiento jurídico considera valiosos y que deben preferirse por sobre la legítima intención de averiguación de la verdad sobre lo ocurrido (Del Río, 2010: 356). Se suele decir que la corroboración judicial de los hechos está sujeta a un marco de limitación institucional (Gascón, 2005: 128).

Estas barreras han sido denominadas reglas contraepistémicas (Gascón, 2005: 128), pues obstan la obtención de conocimiento por parte del sujeto llamado a decidir, lo que impide que alcance un grado mayor de inteligencia e información sobre el asunto (Gascón, 2005: 128). Es decir, aunque exista la posibilidad de acceder a un elemento corroborativo que aporte o contribuya a la averiguación de la verdad, éste no es agregado al proceso - $\mathrm{o}$ en caso de haberlo sido, se excluye-, lo que impide que se pondere al momento de tomar la decisión, pues se considera que la obtención de la verdad o la averiguación de los hechos cede ante otros intereses que la sociedad ha considerado como preponderantes. Esta paradoja de información disponible pero no utilizable no suele presentarse en otros contextos probatorios, como por ejemplo en las ciencias exactas y naturales, sin perjuicio de poder encontrar límites a la actividad de investigación en reglas éticas que regulan las ramas de la ciencia o las agrupaciones de profesionales que las profesan.

En el proceso suelen presentarse, a modo de ejemplo simple, los plazos, periodos $\mathrm{u}$ oportunidades probatorias, entendidos como espacios de tiempo en los cuales una norma fija la rendición de prueba, y que una vez vencidos impiden la agregación o incorporación de dicha pieza de evidencia, no obstante lo significativa que pueda ser, o el alto grado corroborativo sobre los enunciados fácticos que pueda tener. Estas limitaciones suelen ir de la mano de garantías procesales propiamente tales, como la necesidad de un juzgamiento dentro de un tiempo determinado y la finalidad de la decisión judicial.

Dentro de las limitaciones epistémicas más fuertes e importantes, se encuentran las reglas de exclusión de prueba, un conjunto de normas que tiene por objeto garantizar la preponderancia de aquellos valores que se consideran más relevantes que la averiguación de la verdad mediante la exclusión de evidencia que ha sido obtenida con la vulneración de derechos o con la lesión de intereses de alguno de los participantes del proceso o terceros - por ejemplo, a través de la tortura o la violación de 
la privacidad-. La evidencia excluida por estos motivos suele denominarse «prueba ilícita» $\mathrm{o}$ «prueba ilegal», y es posible encontrar regulación orgánica y reconocimiento de ella en muchos ordenamientos jurídicos, paradigmáticamente en la regulación del proceso penal. Una vez que la prueba es excluida, la consecuencia es que el juez no podrá considerar dicho material probatorio para su decisión.

En el contexto deportivo, la existencia de valores preferibles por sobre la averiguación de la verdad por parte del juez parece más difusa. ¿Es, por ejemplo, la celeridad en la decisión una razón suficiente para avanzar en un partido en que se discute si un gol fue marcado en posición adelantada? ¿Puede prescindirse de una tarjeta roja ante una agresión no advertida por el juez, con tal de no detener el partido?

Es indiscutible que se ha hecho un ejercicio de ponderación entre la solución correcta desde el punto de vista de verificación de los hechos y ciertos valores propios de cada deporte que suelen esgrimirse como esenciales para el desarrollo de su actividad, como la continuidad de las acciones para el correcto desempeño del juego - por oposición a las interrupciones que la revisión genera $-{ }^{20} \mathrm{o}$ la misma «esencia» o «pasión» de un determinado deporte. ${ }^{21}$

Dentro de las reglas que constituyen las mencionadas barreras para la averiguación de la verdad en el campo de juego podemos encontrar, por ejemplo, las restricciones que se señalan a los árbitros a través de las limitaciones de competencia que se establecen en la revisión. Se trata de reglas que expresan que la revisión debe ceñirse al motivo o causal que la ha generado, y que impiden que se amplíe la enmienda a otras decisiones que pueden aparecer como manifiestamente erróneas en la propia revisión. Se trata, entonces, de un supuesto en que existe prueba de inmejorable calidad (video de alta definición de múltiples ángulos) que da cuenta de un error del juez y que puede ser determinante en el juego; sin embargo, por no ser el objeto de la revisión solicitada, no puede considerarse. En otras palabras, teniendo toda la información disponible para que la decisión sea revocada y se aplique un fallo correcto - fundado en lo que de verdad ocurrió- dicha información es excluida y no tomada en cuenta.

Ejemplos de lo anterior pueden encontrarse en la regla 13, sección 2 del Rulebook de la NBA, que señala que si se ha solicitado la revisión en video para determinar qué

20. Se sostiene que el VAR supone una desproporción entre la invasión de la continuidad del juego y el aporte a la corrección de las decisiones. Jacob Steinberg, «Officials aim for VAR to bring about $2 \%$ fewer errors by referees», The Guardian, 7 de enero de 2018, disponible en https://bit.ly/32zqGig.

21. En ese sentido, han existido quienes han defendido la subjetividad y falibilidad de los cobros de los jueces como una característica esencial y necesaria del fútbol. Mauricio Pochettino señaló que «el fútbol también es sobre los errores, como nosotros, los jugadores pueden cometer errores, los árbitros cometen errores. Siempre ha sido así y nosotros entendemos el fútbol de esa manera». J. M. Pinochet, «Por qué estoy en contra del VAR, el sistema de videoarbitraje en el fútbol», $B B C$ News, Deportes, 1 de marzo de 2018, disponible en https://bbc.in/2Is1Fyp. 
equipo fue el último en tocar el balón antes de que éste abandonara la cancha, y revisando dicha jugada se detecta la existencia de una infracción de un jugador a otro, ésta no podrá ser cobrada, sin perjuicio de que cobrarla sería la decisión correcta.

Entonces, las reglas de asistencia en video suponen una mejor información para decidir, pero limitadas a través de reglas que permiten su aplicación como asistente en el contexto de verificación de los hechos.

Como en cualquier contexto racional de decisiones, los errores siguen ocurriendo. No hay en la admisión de mecanismos de revisión como el VAR un compromiso absoluto con la eliminación de errores, sino que se introduce un mecanismo y un contexto para disminuirlos asumiendo que la revisión puede encontrarse mejor justificada. Así, en todos los casos en que, durante el juego, no se dé una hipótesis de aplicación de la regla del VAR, aunque tengamos disponible información relevante, no podremos contar con ella por no formar parte de una hipótesis de revisión.

\section{Estándar de prueba}

Una cuestión importante de cara a la administración de la regla de revisión en el VAR es la regla de umbral de suficiencia que la evidencia debe proveer para justificar la adopción de una determinada decisión.

Entendido como el umbral de suficiencia por sobre el cual aceptaremos una hipótesis como probada en juicio (Ferrer, 2007: 139), es decir, como la respuesta a la pregunta sobre cuánta prueba se requiere para probar algo (Zuckerman, 2013: 1.019), el estándar de prueba supone el momento de la decisión probatoria propiamente tal, en el que el juzgador afirma que un enunciado ha quedado o no demostrado a través de la evidencia valorada.

El modelo de estándar de prueba aplicado en revisión de video durante el juego supone establecer un umbral de suficiencia que debe superar la prueba disponible en el caso, el cual - a diferencia de lo que se podría esperar- suele ser bastante alto, incluso más alto que el asociado al proceso penal en ciertos contextos (Calandrillo y Davison, 2017: 24-30). ${ }^{22}$

Así, hasta el año 2016, en la NBA y la NFL el listón de suficiencia que debía cumplirse por la revisión era el de «evidencia incontrovertible» (indisputable visual evidence), ${ }^{23}$ el que dada su configuración suponía que la revisión del video debía permitir que los agentes llamados a decidir pudieran contar con un grado de confirmación casi total, y que la prueba proporcionada por el video no admitiera disputa ni controversia, ${ }^{24}$ es decir, excluyese la duda.

22. El estándar penal sería «más allá de toda duda razonable».

23. 2016 NFL Rulebook, regla 15, sección 2, artículo 5.

24. Jarrett Bell, «At NFL's Command Center, reviews must be right», USA Today, 1o de noviembre de 
Esta regla fue reformada en 2017 en ambas regulaciones, aunque no queda claro que haya sido por considerarla demasiado rigurosa o porque generaba problemas de interpretación, y se estableció el estándar de evidencia clara y conclusiva (clear and obvious visual evidence para la NFL y clear and conclusive evidence para la NBA). ${ }^{25} \mathrm{En}$ alguna medida, se decidió por una cierta admisión de duda que fuese más clara que aquella que se encontraba en la regla original.

La existencia de una regla de estándar explícito en regulaciones de deportes masivos en Estados Unidos puede explicarse por una tradición cultural y jurídica que ha entendido, desde antiguo, la necesidad de fijar un criterio de suficiencia para determinar cuándo y cuándo no los enunciados fácticos deben tenerse por efectivos.

A pesar de ello, el «mundo del fútbol» no ha comprendido — de forma más o menos orgánica- esta misma necesidad. Así, en el protocolo se señala que la revisión de video permitirá cambiar una decisión del árbitro si existe un error claro, que la regulación llama una decisión «manifiestamente incorrecta». ${ }^{26}$ Pero ¿cuándo las pruebas son suficientes para sostener que una decisión ha sido manifiestamente incorrecta? Y por cierto, esa regla, ¿admite, entonces, que las decisiones que sea incorrectas, aunque no manifiestamente, no sean modificadas?

Parece suponer un umbral que debe sobrepasar la evidencia contenida en la revisión, señalando el protocolo que, si no es manifiestamente incorrecta, sino que sólo incorrecta, no se puede cambiar el cobro original. ${ }^{27}$ Esto provocaría que decisiones erróneas - así definidas por la evidencia de la revisión - sean señaladas como tales, pero validadas al mismo tiempo.

¿Cuál es la regla de carga de la prueba aplicable en este ámbito? La respuesta en estas regulaciones es relativamente evidente, pues existe, a nuestro juicio, una construcción normativa que fija una suerte de presunción de corrección o acierto al cobro original durante el desarrollo del juego. El statu quo de continuidad del juego da por correctas todas las decisiones, de modo que su modificación supone el reconocimiento de razones que permitan el cambio de la decisión y la interrupción del juego.

Se establece una distribución de la carga hacia quien desea utilizar el video como asistente, que servirá como regla de desempate en caso de que no se logre una certeza que supere el umbral fijado por el estándar (Ho, 2008: 174), es decir, si no se supera el listón establecido, el cobro no puede revertirse, quedando firme el original. En esta medida, podría ser importante reconocer una hipótesis defendida por quien provoca la revisión: la jugada supone roja directa, o la pelota ha traspasado la línea de gol. De

\footnotetext{
2014, disponible en https://bit.ly/2IwnY $5 \mathrm{~K}$.

25. 2013-2014 NBA Rulebook, regla 13, sección 3.

26. IFAB, «Protocolo...», 4 .

27. IFAB, «Protocolo...», 4.
} 
otro modo, la idea de carga queda bastante inactiva y se generan ciertos problemas de administración de la regla de estándar.

$\mathrm{La}$ "presunción de corrección» a la que se hace referencia podrá encontrar su símil en construcciones inferenciales normativas (González Lagier, 2014: 87-88), como la presunción de inocencia, que para el momento de la toma de decisión opera como una regla de juicio (Valenzuela, 2013: 16), es decir, comanda al sujeto llamado a decidir a inclinarse por favorecer a quien no soportaba dicha carga, favoreciendo en este caso al cobro original en desmedro de la hipótesis alternativa que propicia la revisión. También encontramos un símil en el ámbito administrativo a propósito de la legalidad de los actos administrativos. En definitiva, se trata de la definición de un statu quo que favorece el mantenimiento de las decisiones adoptadas asumiéndolas, a falta de mejores razones, como correctas.

\section{Conclusiones}

El deporte, y en particular el fútbol, es un campo de desarrollo de argumentos probatorios. En la actualidad, la regulación relativa al VAR permite sostener que nos encontramos ante la regulación de un tipo de revisión que permite invalidar una decisión arbitral si se constata un error de ciertas características y en determinadas hipótesis.

Hemos intentado utilizar el VAR para ilustrar conceptos e instituciones propias de la teoría de la prueba a la luz de decisiones en contextos deportivos, de forma de poder ejemplificar la idea expresada en este artículo referida a la actividad probatoria en un contexto reglado.

Con esto, es posible visibilizar la premisa cognoscitivista señalada en los primeros párrafos, que pretende ejemplificar, a través de una de las manifestaciones culturales más masivas y cotidianas - deportes colectivos de alta popularidad (Horne, Tomlinson y Whannel, 2001: 1-10) -, la coincidencia existente entre las normas que regulan la evidencia y la adopción de decisiones que buscan la determinación de lo que ha ocurrido en un caso concreto.

Podemos concluir que, en general, las reglas de prueba de contextos controlados, como son el proceso judicial y los deportes a nivel profesional, suelen regirse por estatutos que, sin ser idénticos, mantienen una similitud suficiente en su aproximación al objeto de dicha regulación, lo que hace posible esta comparación con fines pedagógicos como los que persigue este trabajo.

De esta manera, se ha constatado la existencia de reglas de configuración del objeto de prueba, de la iniciativa o legitimación para solicitar la revisión; la consagración más o menos expresa de reglas contraepistémicas que impiden la revisión y, en consecuencia, la posibilidad de mejorar la decisión, en el sentido de su corrección, fundada en valores deportivos defendidos y tutelados por las reglas de cada deporte; 
así como reglas de distribución de errores y suficiencia probatoria para alterar o confirmar lo que sido sentenciado en tiempo real.

Creemos necesario revisar, en el contexto de la aún incipiente regulación del VAR en el fútbol, las reglas relativas a la iniciativa y carga probatoria y sobre todo la regla de estándar probatorio, de manera de mejorar el escenario de decisión del árbitro, de forma que sean compatibles cuestiones como la continuidad del juego, la fluidez de las acciones, la atención de los espectadores, etcétera, para revestir de mayor asertividad las decisiones que pueden decidir el devenir de un encuentro deportivo, como ocurre en el contexto del proceso judicial.

\section{Referencias}

BAYON, Juan Carlos (2010). «Epistemología, moral y prueba de los hechos: Hacia un enfoque no benthamiano». Revista Mario Alfaro D' Filippo, 2 (4): 6-30. DOI: 10.32997/2256-2796-vol.2-num.4-2010-252.

Calandrillo, Steve y Joseph Davison (2017). «Standards of review in law and sports: How instant replay's asymmetric burdens subvert accuracy and justice». Harvard Journal of Sports \& Entertainment Law, 8: 1-38. Disponible en https://bit. ly/3kq7ucF.

Couture, Eduardo (1981). Fundamentos de derecho procesal civil. Buenos Aires: B de F.

Del Río, Carlos (2010). «Dos formas discutibles de poner en duda el carácter cognoscitivo de la aplicación judicial del derecho penal: El principio del consenso y la garantía de la no agravación punitiva». Revista de Derecho de la Pontificia Universidad Católica de Valparaíso, 34: 349-383. DOI: 10.4067/So718-68512010000100010.

Ferrer, Jordi (2005). Prueba y verdad en el derecho. Barcelona: Marcial Pons.

-. (2007). Valoración racional de la prueba. Barcelona: Marcial Pons.

Fuller, Lon (2002). El caso de los exploradores de cavernas. Buenos Aires: Abeledo Perrot.

GASCón, Marina (2005). «Sobre la posibilidad de formular estándares de prueba objetivos». Cuadernos de Filosofía del Derecho, 28: 127-139. DOI: 10.14198/ DOXA2005.28.10.

González Lagier, Alejandro (2014). «Presunción de inocencia, verdad y objetividad». En Juan García Amado y Pablo Bonorino (coordinadores), Prueba y razonamiento probatorio en derecho: Debates sobre abducción. Granada: Comares.

Hart, Herbert (1994). The concept of law. Oxford: Oxford University Press.

Ho, Hock Lai (2008). A philosophy of evidence law: Justice in the search for truth. Oxford: Oxford University Press.

HoRne, John, Alan Tomlinson y Garry Whannel (2001). Understanding sport: An introduction to the sociological and cultural analysis of sport. Nueva York: Taylor \& Francis. 
KeAne, Adrian y Paul Mckeown (2014). The modern law of evidence. Oxford: Oxford University Press.

Maturana, Cristian y Raúl Montero (2012). Derecho procesal penal. Santiago: Abeledo Perrot.

NaURIGHT, John y Sarah Zipp (2018). «The complex world of global sport». Sport in Society, 21 (8): 1.113-1.119. DOI: 10.1080/17430437.2018.1469846.

Ortells, Manuel (2010). Derecho procesal civil. Pamplona: Thompson-Aranzadi.

RusseL, Jeffrey (2011). "Limitations of the sport-law comparison». Journal of the Philosophy of Sport, 38 (2): 254-272. DOI: 10.1080/00948705.2011.10510425.

SCHAUER, Frederick (2016). «Fuller's fairness: "The case of the Speluncean explorers"». University of Queensland Law Journal, 35 (1): 11-12. Disponible en https:// bit.ly/3kjPZuH.

Taruffo, Michele (2008). La prueba. Barcelona: Marcial Pons.

VAlenzuela, Jonatan (2017). «Sobre el estándar de prueba cautelar: Decisiones provisionales y actividad probatoria en el proceso penal». En Hechos, pena y proceso. Santiago: Rubicón.

-. (2013). «Inocencia y razonamiento probatorio». Revista de Estudios de la Justicia, 18: 13-23. DOI: 10.5354/0718-4735.2013.29907.

Zuckermann, Adrian (2013). Zuckerman on civil procedure, principles and practice. Londres: Sweet and Maxwell.

\section{Sobre los autores}

Jesús Ezurmendia Álvarez es abogado. Licenciado en Ciencias Jurídicas y Sociales por la Universidad de Chile. Magister en Derecho (LLM) University College of London, Reino Unido. Profesor asistente de Derecho Procesal, Facultad de Derecho, Universidad de Chile. Su correo electrónico es jezurmendia@derecho.uchile.cl. (iD) https://orcid.org/o0oo-0002-0616-2823.

Jonatan Valenzuela Saldías es abogado. Licenciado en Ciencias Jurídicas y Sociales por la Universidad de Chile. Doctor en Derecho por la Universidad de Gerona, España. Profesor asociado de Derecho Procesal, Facultad de Derecho, Universidad de Chile. Su correo electrónico es jvalenzuela@derecho.uchile.cl. (D) https:// orcid.org/00oo-0002-9792-5637. 


\title{
REVISTA CHILENA DE DERECHO Y TECNOLOGÍA
}

La Revista de Chilena de Derecho y Tecnología es una publicación académica semestral del Centro de Estudios en Derecho Informático de la Facultad de Derecho de la Universidad de Chile, que tiene por objeto difundir en la comunidad jurídica los elementos necesarios para analizar y comprender los alcances y efectos que el desarrollo tecnológico y cultural han producido en la sociedad, especialmente su impacto en la ciencia jurídica.

\author{
EDITOR GENERAL \\ Daniel Álvarez Valenzuela \\ (dalvarez@derecho.uchile.cl) \\ SITIO WEB \\ rchdt.uchile.cl \\ CORREO ELECTRÓNICO \\ rchdt@derecho.uchile.cl \\ LICENCIA DE ESTE ARTÍCULO \\ Creative Commons Atribución Compartir Igual 4.o Internacional
}

\begin{abstract}
La edición de textos, el diseño editorial
y la conversión a formatos electrónicos de este artículo

estuvieron a cargo de Tipográfica

(www.tipografica.io).
\end{abstract}

